Pacific

Journal of

Mathematics

A LIOUVILLE TYPE THEOREM FOR A GENERAL CLASS OF OPERATORS ON COMPLETE MANIFOLDS

Marco Rigoli, Maura Salvatori, and Marco Vignati

Volume $194 \quad$ No. 2

June 2000 


\title{
A LIOUVILLE TYPE THEOREM FOR A GENERAL CLASS OF OPERATORS ON COMPLETE MANIFOLDS
}

\author{
Marco Rigoli, Maura Salvatori, and Marco Vignati
}

We prove a weak maximum principle and some Liouville type theorems for a general class of operators on complete manifolds under appropriate volume growth conditions.

\section{Introduction.}

Let $(M,\langle\rangle)$ be a complete Riemannian manifold, and, for a fixed reference point $o \in M$, set $r(x)=\operatorname{dist}_{(M,\langle\rangle)}(x, o)$. Thus $B_{R}$ and $\partial B_{R}$ denote, respectively, the geodesic ball and sphere of radius $R$ centered at $o$. In what follows we shall always tacitly assume $M$ connected. With $\phi$ we indicate a real function satisfying the following requirements

$$
\begin{cases}\text { i) } \quad \phi \in \mathcal{C}^{1}((0,+\infty)) \cap \mathcal{C}^{0}([0,+\infty)) \\ \text { ii) } \phi(0)=0, \phi(t)>0 \text { on }(0,+\infty) \\ \text { iii) } \phi(t) \leq A t^{\delta} \quad \text { on }[0,+\infty) \quad \text { for some constants } A, \delta>0\end{cases}
$$

We state our main result in the form of the following Liouville type:

Theorem A. Let $(M,\langle\rangle), r(x)$ be as above and let $\phi$ satisfy (0.1). Let $u \in \mathcal{C}^{2}(M)$ be a solution of the equation

$$
\operatorname{div}\left(|\nabla u|^{-1} \phi(|\nabla u|) \nabla u\right)=a
$$

for some $a \in \mathbb{R}$, such that

$$
u(x)=o(\log r(x)) \quad \text { as } r(x) \rightarrow+\infty .
$$

Then $u$ is constant (and $a=0$ ) provided

$$
\operatorname{vol}\left(\partial B_{R}\right) \leq c R^{\delta} \quad \text { for } R \gg 1
$$

for some $c>0$, where $\delta$ is as in (0.1) iii).

For instance, the choices

$$
\phi(t)=\frac{t}{\left(1+t^{2}\right)^{\alpha}}, \alpha>0 ; \quad \phi(t)=t^{p-1}, p>1 ;
$$

on the left hand side of (0.2) yield, respectively, the generalized mean curvature operator and the $p$-Laplacian. In these cases condition $(0.1)$ is certainly satisfied with the choices $\delta=1$ and $\delta=p-1$ respectively, and some positive A. 
In order to prove Theorem A we need two results, Theorems 1 and 3, of independent interest. Theorem 1 is proven in the next section; Theorem 3 is stated in Section 3 and used to prove the main result, while its proof is contained in Section 4. Comments on the mean curvature operator and the $p$-Laplacian are contained in Sections 2 and 5, respectively.

\section{Theorem 1 and its proof.}

The following result can be considered as a weak maximum principle in the spirit of the well known result of Omori and Yau for the Laplace-Beltrami operator, see $[\mathbf{C Y}]$. Curvature conditions are here replaced by the volume growth assumption (1.1) below.

Theorem 1. Let $(M,\langle\rangle)$ be a complete manifold, $r(x)=\operatorname{dist}_{(M,\langle\rangle)}(x, o)$ and $\phi$ as in (0.1). Suppose that for some constants $c>0, n \geq 1+\delta$ and $\delta$ as in (0.1) iii) we have

$$
\operatorname{vol}\left(B_{R}\right) \leq c R^{n} \quad \text { for } R \gg 1 .
$$

Let $u$ be a $\mathcal{C}^{2}$ function on $M$ such that

$$
\limsup _{r(x) \rightarrow+\infty} \frac{u(x)}{r(x)^{\frac{\delta+1}{\delta}}}=b<+\infty .
$$

Then,

$$
\operatorname{Inf}_{M} \operatorname{div}\left(|\nabla u|^{-1} \phi(|\nabla u|) \nabla u\right) \leq A n\left(b^{*} \frac{1+\delta}{\delta}\right)^{\delta}
$$

with $A, \delta$ as in (0.1) iii) and $b^{*}=\max (b, 0)$.

In the proof of the Theorem we shall make use of the following Lemma from calculus:

Lemma. Let $\delta, \alpha, \beta, \sigma>0$ be fixed constants and let $g:[0,+\infty) \rightarrow \mathbb{R}$ be the function

$$
g(x)=\alpha x^{\frac{1+\delta}{\delta}}-\beta x+\sigma .
$$

If

$$
\Lambda \leq \alpha-\frac{\beta^{\frac{1+\delta}{\delta}} \delta}{(1+\delta)^{\frac{1+\delta}{\delta}} \sigma^{1 / \delta}}
$$

then

$$
g(x) \geq \Lambda x^{\frac{1+\delta}{\delta}} \quad \text { on }[0,+\infty) .
$$

Proof. The function $f(x)=g(x)-\Lambda x^{\frac{1+\delta}{\delta}}$ has an absolute minimum either at 0 with value $\sigma>0$ or at

$$
x_{0}=\left[\frac{\beta \delta}{(\alpha-\Lambda)(1+\delta)}\right]^{\delta} .
$$


However, (1.4) implies $f\left(x_{0}\right) \geq 0$ and we obtain the desired result.

Proof of Theorem 1. Fix $B>b^{*}$; the theorem will be proven once we show that

$$
\operatorname{Inf}_{M} \operatorname{div}\left(|\nabla u|^{-1} \phi(|\nabla u|) \nabla u\right) \leq k n,
$$

where, for convenience, we have set

$$
k=A B^{\delta}\left(\frac{1+\delta}{\delta}\right)^{\delta} .
$$

In order to do this we reason by contradiction, and we suppose that, for some $\epsilon>0$,

$$
\operatorname{Inf}_{M} \operatorname{div}\left(|\nabla u|^{-1} \phi(|\nabla u|) \nabla u\right)=(k+\epsilon) n .
$$

Because of (1.2), there exists $R_{0}>0$ such that

$$
u(x) \leq B r(x)^{\frac{1+\delta}{\delta}} \quad \text { on } M \backslash B_{R_{0}},
$$

so that the function

$$
v(x)=B\left(\frac{1+\delta}{\delta}\right) r(x)^{\frac{1+\delta}{\delta}}-u(x)
$$

satisfies

$$
v(x) \geq \frac{B}{\delta} r(x)^{\frac{1+\delta}{\delta}}
$$

on $M \backslash B_{R_{0}}$. However, (1.2) and (1.6) are independent of additive constants, so that we may assume that (1.8) holds on $M$, and $v(o)>0$.

For $R>0$, let $g \in \mathcal{C}^{\infty}(M)$ satisfy:

$$
\begin{cases}\text { i) } 0 \leq g \leq 1 ; & \text { ii) } g \equiv 1 \text { on } B_{R} \\ \text { iii) } g \equiv 0 \text { outside } B_{2 R} ; & \text { iv) }|\nabla g| \leq \frac{C}{R} \text { on } B_{2 R} \backslash B_{R} .\end{cases}
$$

Using $p>1$ as a parameter (to be specified later) and the cut-off function $g$, we define the vector field

$$
W=g^{1+\delta} v^{1-p}|\nabla u|^{-1} \phi(|\nabla u|) \nabla u .
$$

Then, from (1.7)

$$
\begin{aligned}
\operatorname{div} W= & (1+\delta) g^{\delta} v^{1-p}|\nabla u|^{-1} \phi(|\nabla u|)\langle\nabla g, \nabla u\rangle \\
& +g^{1+\delta} v^{-p}\left[v \operatorname{div}\left(|\nabla u|^{-1} \phi(|\nabla u|) \nabla u\right)+(p-1)|\nabla u| \phi(|\nabla u|)\right. \\
& \left.-B\left(\frac{1+\delta}{\delta}\right)^{2}(p-1) r^{1 / \delta}|\nabla u|^{-1} \phi(|\nabla u|)\langle\nabla u, \nabla r\rangle\right] .
\end{aligned}
$$

Using (0.1), (1.6), (1.8) and the inequality

$$
|\langle\nabla u, \nabla r\rangle| \leq|\nabla u|,
$$


from the above we obtain

$$
\begin{aligned}
\operatorname{div} W \geq & (1+\delta) g^{\delta} v^{1-p}|\nabla u|^{-1} \phi(|\nabla u|)\langle\nabla g, \nabla u\rangle \\
& +g^{1+\delta} v^{-p}\left[(p-1) A^{-1 / \delta} \phi(|\nabla u|)^{\frac{1+\delta}{\delta}}\right. \\
& \left.-B(p-1)\left(\frac{1+\delta}{\delta}\right)^{2} r^{1 / \delta} \phi(|\nabla u|)+\frac{B}{\delta}(k+\epsilon) n r^{\frac{1+\delta}{\delta}}\right]
\end{aligned}
$$

on $M$. We apply the Lemma respectively with the choices $x=\phi(|\nabla u|)$, $\alpha=(p-1) A^{-1 / \delta}, \beta=B(p-1)\left(\frac{1+\delta}{\delta}\right)^{2} r^{1 / \delta}, \sigma=\frac{B}{\delta}(k+\epsilon) n r^{\frac{1+\delta}{\delta}}$ to deduce that, for

$$
\Lambda=(p-1)\left[A^{-1 / \delta}-B\left(\frac{1+\delta}{\delta}\right)^{\frac{1+\delta}{\delta}}\left(\frac{p-1}{(k+\epsilon) n}\right)^{1 / \delta}\right]
$$

we have

$$
\operatorname{div} W \geq(1+\delta) g^{\delta} v^{1-p}|\nabla u|^{-1} \phi(|\nabla u|)\langle\nabla g, \nabla u\rangle+\Lambda g^{1+\delta} v^{-p} \phi(|\nabla u|)^{\frac{1+\delta}{\delta}}
$$

on $M$. An immediate check shows that $\Lambda>0$ if and only if

$$
p-1<\frac{\delta n}{1+\delta}\left(1+\frac{\epsilon}{k}\right) .
$$

The divergence theorem applied to (1.9) yields

$$
\Lambda \int_{B_{2 R}} g^{1+\delta} v^{-p} \phi(|\nabla u|)^{\frac{1+\delta}{\delta}} \leq(1+\delta) \int_{B_{2 R}} g^{\delta} v^{1-p} \phi(|\nabla u|)|\nabla g|,
$$

and then we apply Hölder's inequality to the right hand side, with conjugate exponents $(1+\delta) / \delta$ and $(1+\delta)$, to obtain

$$
\begin{aligned}
& \int_{B_{2 R}} g^{\delta} v^{1-p} \phi(|\nabla u|)|\nabla g|=\int_{B_{2 R}}\left[g^{\delta} v^{-\frac{p \delta}{1+\delta}} \phi(|\nabla u|)\right]\left[v^{1-\frac{p}{1+\delta}}|\nabla g|\right] \\
& \leq\left\{\int_{B_{2 R}} g^{1+\delta} v^{-p} \phi(|\nabla u|)^{\frac{1+\delta}{\delta}}\right\}^{\frac{\delta}{1+\delta}}\left\{\int_{B_{2 R}} v^{1+\delta-p}|\nabla g|^{1+\delta}\right\}^{\frac{1}{1+\delta}} .
\end{aligned}
$$

In other words,

$$
\begin{aligned}
& \Lambda \int_{B_{2 R}} g^{1+\delta} v^{-p} \phi(|\nabla u|)^{\frac{1+\delta}{\delta}} \\
& \leq(1+\delta)\left\{\int_{B_{2 R}} g^{1+\delta} v^{-p} \phi(|\nabla u|)^{\frac{1+\delta}{\delta}}\right\}^{\frac{\delta}{1+\delta}}\left\{\int_{B_{2 R}} v^{1+\delta-p}|\nabla g|^{1+\delta}\right\}^{\frac{1}{1+\delta}},
\end{aligned}
$$

whence

$$
\left(\frac{\Lambda}{1+\delta}\right)^{1+\delta} \int_{B_{2 R}} g^{1+\delta} v^{-p} \phi(|\nabla u|)^{\frac{1+\delta}{\delta}} \leq \int_{B_{2 R}} v^{1+\delta-p}|\nabla g|^{1+\delta} .
$$


Thus, using the properties of the cut-off function $g$,

$$
\left(\frac{\Lambda}{1+\delta}\right)^{1+\delta} \int_{B_{R}} v^{-p} \phi(|\nabla u|)^{\frac{1+\delta}{\delta}} \leq \frac{c}{R^{1+\delta}} \operatorname{vol}\left(B_{2 R}\right) \operatorname{Sup}_{B_{2 R} \backslash B_{R}} v^{1+\delta-p}
$$

for some absolute constant $c>0$.

Using (1.1) and (1.8) and assuming

$$
p-1 \geq \delta
$$

we have

$$
\left(\frac{\Lambda}{1+\delta}\right)^{1+\delta} \int_{B_{R}} v^{-p} \phi(|\nabla u|)^{\frac{1+\delta}{\delta}} \leq \widetilde{c} R^{\frac{1+\delta}{\delta}(1+\delta-p)+n-(1+\delta)}
$$

for some constant $\widetilde{c}>0$. If we can guarantee that

$$
n+\frac{1+\delta}{\delta}(1+\delta-p)-(1+\delta)<0,
$$

from the property (0.1) ii) we obtain $|\nabla u| \equiv 0$ on $M$, i.e., $u$ constant, contradicting (1.6).

The proof of (1.5) will then be accomplished once we show that it is possible to choose $p$ to satisfy (1.10), (1.12) and (1.13), that is,

$$
\left\{\begin{array}{l}
p-1<\frac{\delta n}{1+\delta}\left(1+\frac{\epsilon}{k}\right) \\
p-1 \geq \delta \\
p-1>\frac{n \delta}{1+\delta}
\end{array}\right.
$$

These conditions are compatible, since $n \geq 1+\delta$ and $\epsilon>0$. Thus, (1.5) holds, and letting $B \rightarrow b^{*}$ we obtain (1.3).

\section{Comments on Theorem 1.}

Estimate (1.3) of Theorem 1 is sharp. Indeed, let $(M,\langle\rangle)=,\left(\mathbb{R}^{n},\langle\rangle,\right)$ be Euclidean space with its canonical metric. Choose $\phi(t)=t$ so that the operator we are considering is the Laplace-Beltrami operator $\Delta$. Let

$$
u(x)=b|x|^{2}
$$

where $|x|=\operatorname{dist}_{\left(\mathbb{R}^{n},\langle,\rangle\right)}(x, 0)$ for some $b>0$. Then

$$
\Delta u=2 b n
$$

and this perfectly agrees with (1.3) where in the present case we have to choose $A=1, \delta=1$.

Theorem 1 is based on the assumption that $(M,\langle\rangle$,$) has at most poly-$ nomial growth, that is (1.1) holds. We now show that this condition is necessary for Theorem 1 to hold. 
Let $(M,\langle\rangle)=,\left(\mathbb{H}^{m},\langle\rangle,\right)$ be hyperbolic $m$-dimensional space, $m \geq 2$, with its canonical metric of constant negative curvature -1 . We realize $\mathbb{H}^{m} \backslash$ $\{0\}$ in polar coordinates $(r, \vartheta) \in(0,+\infty) \times S^{m-1}$ and its metric $\langle$,$\rangle as$

$$
\langle,\rangle=d r^{2}+(\mathrm{Sh} r)^{2} d \vartheta^{2}
$$

with $d \vartheta^{2}$ the standard metric on $S^{m-1}$. One easily verifies that

$$
u(x)=\int_{0}^{r(x)}(\operatorname{Sh} t)^{-(m-1)}\left(\int_{0}^{t}(\operatorname{Sh} s)^{m-1} d s\right) d t
$$

is $\mathcal{C}^{2}\left(\mathbb{H}^{m}\right)$ and satisfies

$$
\Delta u \equiv 1
$$

Furthermore,

$$
u(x) \sim r(x) \quad \text { as } r(x) \rightarrow+\infty .
$$

Thus, (1.2) is met with $b=0$, but conclusion (1.3) is violated. (Here and in the sequel the expression $A(t) \sim B(t)$ as $t \rightarrow t_{0}$ means that $A(t) / B(t)$ is bounded and remains away from zero as $t \rightarrow t_{0}$.)

However, it is possible to give a version of the theorem in case $(M,\langle\rangle$, has at most exponential growth. We state the result with no proof, and remind that the case of the Laplace operator has been first obtained by L. Karp, [K1].

Theorem 2. Let $(M,\langle\rangle$,$) be a complete manifold, r(x)=|x|=$ $\operatorname{dist}_{(M,\langle,\rangle)}(x, o)$ and $\phi$ as in (0.1) with $\delta \geq 1$. Suppose that for some constant $\gamma>0$

$$
\log \left(\operatorname{vol}\left(B_{R}\right)\right) \leq \gamma R \quad \text { for } R \gg 1 .
$$

Let $u \in \mathcal{C}^{2}(M)$ satisfy

$$
\limsup _{r(x) \rightarrow+\infty} \frac{u(x)}{r(x)}=b<+\infty
$$

Then,

$$
\operatorname{Inf}_{M} \operatorname{div}\left(|\nabla u|^{-1} \phi(|\nabla u|) \nabla u\right) \leq \frac{A \gamma 2^{1+\delta}(b \delta)^{\delta}}{(1+\delta)^{1+\delta}}
$$

with $A, \delta$ as in (0.1) iii).

This result can be applied to the following geometrical problem.

Given $(M,\langle\rangle$,$) and a smooth function u: M \rightarrow \mathbb{R}$ we associate to $u$ the graph $\Gamma_{u}: M \rightarrow M \times \mathbb{R}$ defined by

$$
\Gamma_{u}: x \rightarrow(x, u(x)) .
$$

Indicating with $($, ) the product metric on $M \times \mathbb{R}$,

$$
\Gamma_{u}:\left(M, \Gamma_{u}^{*}(,)\right) \rightarrow(M \times \mathbb{R},(,))
$$


becomes an isometric embedding. Let $\nabla$, div, $|\cdot|$ denote the gradient, the divergence operator and the norm with respect to the metric $\langle$,$\rangle . Then \Gamma_{u}$ has constant mean curvature $\frac{a}{m}$ if and only if

$$
\operatorname{div}\left(\frac{\nabla u}{\sqrt{1+|\nabla u|^{2}}}\right)=a \quad \text { on } M
$$

for some $a \in \mathbb{R}$. If $a=0, \Gamma_{u}$ is a minimal graph.

In case $M=\mathbb{R}^{m}$ Euclidean space, a well known result of Heinz, $[\mathbf{H}]$, for surfaces, generalized by Flanders, $[\mathbf{F}]$, and Chern, $[\mathbf{C}]$, to any dimension, implies that a graph on $\mathbb{R}^{m}$ with constant mean curvature is necessarily a minimal graph.

Indeed, a nice observation of Salavessa, [S], shows that if $h(M)$ is the Cheeger constant of $M$ and $H_{\Gamma}$ is the mean curvature of a constant mean curvature graph then

$$
\left|H_{\Gamma}\right| \leq \frac{1}{m} h(M) .
$$

Since $h\left(\mathbb{R}^{m}\right)=0$ we deduce the validity of the above conclusion.

We observe that the hyperbolic space $\mathbb{H}^{m}$ has constant sectional curvature -1 , and $h\left(\mathbb{H}^{m}\right)=m-1$; in this case there do exist nonminimal graphs with constant mean curvature.

For instance, realizing $\mathbb{H}^{m}$ in polar coordinates as in the remark above, it is not hard to verify that, having chosen a constant $a \in(0, m-1]$, the smooth function

$$
u(x)=\int_{0}^{r(x)} \frac{(\operatorname{Sh} t)^{1-m} \int_{0}^{t} a(\operatorname{Sh} s)^{m-1} d s}{\left\{1-(\operatorname{Sh} t)^{2(1-m)}\left[\int_{0}^{t} a(\operatorname{Sh} s)^{m-1} d s\right]^{2}\right\}^{1 / 2}} d t
$$

defines a graph $\Gamma_{u}: \mathbb{H}^{m} \rightarrow \mathbb{H}^{m} \times \mathbb{R}$ with constant mean curvature $a$.

We notice that, in this example,

$$
u(x) \sim r(x) \quad \text { as } r(x) \rightarrow+\infty ;
$$

thus, in order to obtain a result similar to that of Heinz, Chern and Flanders when $(M,\langle\rangle$,$) growths exponentially, it seems natural to require some$ growth condition on $u$. Considering, if necessary, $v=-u$, we can, without loss of generality, assume $a \geq 0$. According to Theorem 2 we have:

Corollary. Let $(M,\langle\rangle$,$) be a complete manifold as in Theorem 2. Let \Gamma_{u}$ : $M \rightarrow M \times \mathbb{R}$ be a graph with constant mean curvature such that

$$
u(x)=o(r(x)) \quad \text { as } r(x) \rightarrow+\infty .
$$

Then $\Gamma_{u}$ is minimal. 
Remark. The example above shows that assumption (2.2) is optimal for the Corollary to hold true.

\section{Proof of Theorem A.}

It is well known that complete manifolds of moderate volume growth are parabolic. The next result can be considered a generalization of this fact. However, we observe that, in case of the Laplace-Beltrami operator, (3.1) below becomes $\frac{1}{\operatorname{vol}\left(\partial B_{r}(o)\right)} \notin L^{1}(+\infty)$ and it is not obvious that this condition implies moderate volume growth in the sense of $[\mathbf{K 2}]$ without further requirements.

Theorem 3. Let $(M,\langle\rangle)$ be a complete manifold, $r(x)=\operatorname{dist}_{(M,\langle\rangle)}(x, o)$ and $\phi$ as in (0.1). Assume, with $\delta>0$ as in (0.1) iii), that

$$
\frac{1}{\operatorname{vol}\left(\partial B_{r}(o)\right)^{1 / \delta}} \notin L^{1}(+\infty) \text {. }
$$

Let $u \in \mathcal{C}^{2}(M)$ be a solution of the differential inequality

$$
\operatorname{div}\left(|\nabla u|^{-1} \phi(|\nabla u|) \nabla u\right) \geq 0 \quad \text { on } M .
$$

Then $u$ is constant provided

$$
\liminf _{r \rightarrow+\infty}\left(\operatorname{Sup}_{B_{r}} u\right)\left(\int_{1}^{r} \frac{d t}{\operatorname{vol}\left(\partial B_{t}(o)\right)^{1 / \delta}}\right)^{-1}=0 .
$$

Remark. Condition (3.1) tells that the volume of $B_{r}(o)$ increases "slowly" with the radius. If this condition is not met, requirement (3.3) is not translation invariant, while (3.2) and the conclusion of the theorem do not depend on additive constants. Hence, (3.1) is necessary to give meaning to the statement.

Proof of Theorem A. Let $u \in \mathcal{C}^{2}(M)$ satisfy (0.2). As we did before, we may assume $a \geq 0$, and we first prove that $a=0$. Because of $(0.4)$ we see that (1.1) of Theorem 1 is satisfied with $n=1+\delta$. While, because of $(0.3),(1.2)$ is satisfied with $b=0$. We then deduce from Theorem 1 that

$$
a=\operatorname{Inf}_{m} \operatorname{div}\left(|\nabla u|^{-1} \phi(|\nabla u|) \nabla u\right) \leq 0
$$

that is, $a=0$.

Next, from (0.4),

$$
\frac{1}{\operatorname{vol}\left(\partial B_{r}\right)^{1 / \delta}} \geq \frac{1}{r} \notin L^{1}(+\infty)
$$

Thus (3.3) of Theorem 3 is satisfied because of (0.3). Applying Theorem 3 we deduce that $u$ is constant. 


\section{Proof of Theorem 3.}

The proof of Theorem 3 has been inspired by some recent result of J.F. Hwang, [H1] Theorem 3, and [H2] Theorem 2.2; which in turn is closely related to Collin and Krust, $[\mathbf{C K}]$, and Mikljukov, $[\mathbf{M}]$. Nevertheless, there are some relevant differences. First in $[\mathbf{H 1}],[\mathbf{C K}],[\mathbf{M}],[\mathbf{H 2}]$ the attention is basically focused on the mean curvature operator on $\mathbb{R}^{2}$; secondly the general class of operators considered in Theorem 4 of $[\mathbf{H 1}]$ and Theorem 5.1 of [H2] (both stated without proofs) differs from our even on $\mathbb{R}^{2}$; notably it does not contain the $p$-Laplace operator for $p \neq 2$.

Proof of Theorem 3. We reason by contradiction, and assume that $u$ is nonconstant. We choose a regular value $a$ of $u$ such that, having set

$$
\Omega=\{x \in M: u(x)>a\},
$$

$\partial \Omega$ is a non-empty hypersurface in $M$. Let $R_{0}=\operatorname{dist}(o, \bar{\Omega})$ and, for $r>R_{0}$, define

$$
\begin{gathered}
\Omega_{r}=\Omega \cap B_{r}(o) \\
F_{r}=\partial B_{r}(o) \cap \Omega ; \quad \widetilde{F}_{r}=\partial \Omega_{r} \backslash F_{r} .
\end{gathered}
$$

As we pointed out in the remark following the statement of Theorem 3 , the validity of (3.1) forces (3.3) to be independent of additive constants. Hence, we prove the theorem for the function $v=u-a$ or, simpler, we assume $a=0$. With these assumptions, $u$ satisfies (3.2) and (3.3), and is strictly positive in $\Omega$. We set

$$
\begin{gathered}
h(r)=\int_{F_{r}}|\nabla u| \phi(|\nabla u|) \\
H(r)=\int_{\Omega_{r}}|\nabla u| \phi(|\nabla u|)=\int_{R_{0}}^{r} h(t) d t .
\end{gathered}
$$

Since $|\nabla u| \neq 0$ on $\partial \Omega$, the set $\Omega_{r}$ is open, and $H(r)>0$, for $r>R_{0}$.

Our first aim is to obtain an estimate from above for $H$. In order to do this, we consider the vector field

$$
W=u|\nabla u|^{-1} \phi(|\nabla u|) \nabla u \quad \text { on } M .
$$

If $\nu$ is the outward unit vector normal to $\partial \Omega_{t}, t \geq R_{0}$, we define

$$
\rho(t)=\int_{F_{t}}\langle W, \nu\rangle .
$$

Using Hölder's inequality with conjugate exponents $1+\delta$ and $1+1 / \delta$, we obtain

$$
\rho(t) \leq\left(\operatorname{Sup}_{F_{t}} u\right)\left(\operatorname{vol}\left(F_{t}\right)\right)^{\frac{1}{1+\delta}}\left\{\int_{F_{t}} \phi(|\nabla u|)^{\frac{1+\delta}{\delta}}\right\}^{\frac{\delta}{1+\delta}} \quad \text { for } t \geq R_{0}
$$


so that, from (4.1) and (0.1) iii),

$$
\rho(t) \leq c \operatorname{vol}\left(F_{t}\right)^{\frac{1}{1+\delta}} h(t)^{\frac{\delta}{1+\delta}} \operatorname{Sup}_{F_{t}} u \quad \text { for } t \geq R_{0},
$$

and some constant $c>0$. On the other hand, (3.2), the divergence theorem and the fact that $u$ vanishes on $\widetilde{F}_{t}$ yield

$$
\rho(t) \geq H(t),
$$

whence $\operatorname{vol}\left(F_{t}\right)>0$ for $t>R_{0}$. Thus, if $r \geq R>R_{0}$, we have, from (4.2), (4.4) and the above inequality

$$
\begin{aligned}
& \frac{1}{H(R)^{1 / \delta}} \geq \frac{1}{H(R)^{1 / \delta}}-\frac{1}{H(r)^{1 / \delta}}=\frac{1}{\delta} \int_{R}^{r} \frac{h(t)}{H(t)^{1+1 / \delta}} d t \\
& \geq \frac{1}{\delta} \int_{R}^{r} \frac{h(t)}{\rho(t)^{1+1 / \delta}} d t \\
& \geq c \int_{R}^{r} \frac{d t}{\left(\operatorname{Sup}_{F_{t}} u\right)^{1+1 / \delta} \operatorname{vol}\left(F_{t}\right)^{1 / \delta}}
\end{aligned}
$$

that is the desired upper estimate on $H$ :

$$
\frac{1}{H(R)} \geq C\left(\int_{R}^{r} \frac{d t}{\left(\operatorname{Sup}_{F_{t}} u\right)^{1+1 / \delta} \operatorname{vol}\left(F_{t}\right)^{1 / \delta}}\right)^{\delta}
$$

valid for $r \geq R>R_{0}$.

Next, we obtain a lower estimate for $H$. In order to do this, we choose a $\mathcal{C}^{1}$ function $\alpha: \mathbb{R} \rightarrow \mathbb{R}$ such that

(4.6) i) $\alpha(0)=0$;

ii) $\alpha^{\prime}(t)>0$ on $(0,+\infty)$;

iii) $0<\operatorname{Sup} \alpha=L<+\infty$;

and we consider the vector field

$$
Z=\alpha(u)|\nabla u|^{-1} \phi(|\nabla u|) \nabla u .
$$

With the same choice of $\nu$ as above, we define

$$
\gamma(t)=\int_{F_{t}}\langle Z, \nu\rangle
$$

By (4.6) i), $Z$ vanishes on $\widetilde{F}_{t}$, and the divergence theorem yields

$$
\begin{aligned}
\gamma(t) & =\int_{\Omega_{t}} \operatorname{div} Z \\
& =\int_{\Omega_{t}} \alpha(u) \operatorname{div}\left(|\nabla u|^{-1} \phi(|\nabla u|) \nabla u\right)+\int_{\Omega_{t}} \alpha^{\prime}(u) \phi(|\nabla u|)|\nabla u| .
\end{aligned}
$$


Hence, using (4.6) and (3.2), we deduce that $\gamma$ is non-negative and nondecreasing. More precisely, it is possible to find $\bar{R}>R_{0}$ and $C>0$ such that

$$
C \leq \gamma(t) \leq L \int_{F_{t}} \phi(|\nabla u|) \quad \text { for each } t \geq \bar{R} .
$$

Notice that the second inequality is due to (4.6) iii) and holds for $t \geq R_{0}$, while the first one is a consequence of the fact that $u$ is non-constant on $\Omega$ (hence, $\gamma(t) \not \equiv 0$ on $\left[R_{0},+\infty\right)$ ). The double inequality (4.7) can be used to estimate from below the function $H$. Indeed, applying Hölder's inequality and (4.7) to (4.1) we get

$$
h(t) \geq \frac{c}{\operatorname{vol}\left(F_{t}\right)^{1 / \delta}} \quad \text { for } t \geq \bar{R}
$$

whence

$$
H(s)-H(S) \geq c \int_{S}^{s} \frac{d t}{\operatorname{vol}\left(F_{t}\right)^{1 / \delta}} \quad \text { for } s \geq S \geq \bar{R} .
$$

We shall use both (4.5) and (4.8) in the form

$$
\frac{1}{H(R)} \geq c\left(\int_{R}^{r} \frac{d t}{\left(\operatorname{Sup}_{F_{t}} u\right)^{1+1 / \delta} \operatorname{vol}\left(F_{t}\right)^{1 / \delta}}\right)^{\delta}
$$

$$
H(R)-H(\bar{R}) \geq c \int_{\bar{R}}^{R} \frac{d t}{\operatorname{vol}\left(F_{t}\right)^{1 / \delta}}
$$

for $r>R \geq \bar{R}$. In order to contradict assumption (3.3), we first notice that, if $B$ is any fixed positive constant, the equation

$$
x^{1+\delta}-B x^{\delta}+\frac{\delta^{\delta}}{(1+\delta)^{1+\delta}} B^{1+\delta}=0
$$

has exactly one solution in the interval $(0, B)$, and this solution is $x_{0}=\frac{\delta}{1+\delta} B$. For $r>\bar{R}$ fixed, we set

$$
B=\int_{\bar{R}}^{r} \frac{d t}{\operatorname{vol}\left(F_{t}\right)^{1 / \delta}}
$$

thus, there exists a unique $R=R(r)$, with $\bar{R}<R<r$, such that

$$
x_{0}=\frac{\delta}{1+\delta} B=\int_{R}^{r} \frac{d t}{\operatorname{vol}\left(F_{t}\right)^{1 / \delta}},
$$


and (4.11) becomes

$$
\left(\int_{R}^{r} \frac{d t}{\operatorname{vol}\left(F_{t}\right)^{1 / \delta}}\right)^{\delta}\left(\int_{\bar{R}}^{R} \frac{d t}{\operatorname{vol}\left(F_{t}\right)^{1 / \delta}}\right)=\frac{\delta^{\delta}}{(1+\delta)^{1+\delta}}\left(\int_{\bar{R}}^{r} \frac{d t}{\operatorname{vol}\left(F_{t}\right)^{1 / \delta}}\right)^{1+\delta} .
$$

Combining (4.9), (4.10) and this last inequality we obtain

$$
\begin{aligned}
1 & \geq \frac{H(R)-H(\bar{R})}{H(R)} \\
& \geq \frac{c}{\left(\operatorname{Sup}_{B_{r}} u\right)^{1+\delta}}\left(\int_{R}^{r} \frac{d t}{\operatorname{vol}\left(F_{t}\right)^{1 / \delta}}\right)^{\delta}\left(\int_{\bar{R}}^{R} \frac{d t}{\operatorname{vol}\left(F_{t}\right)^{1 / \delta}}\right) \\
& =\frac{c}{\left(\operatorname{Sup}_{B_{r}} u\right)^{1+\delta}}\left(\int_{\bar{R}}^{r} \frac{d t}{\operatorname{vol}\left(F_{t}\right)^{1 / \delta}}\right)^{1+\delta} \\
& \geq\left(\frac{\int_{\bar{R}}^{r} \frac{d t}{\operatorname{vol}\left(F_{t}\right)^{1 / \delta}}}{\operatorname{Sup}_{B_{t}}}\right)^{1+\delta}
\end{aligned}
$$

valid for $r>\bar{R}$ fixed, $R=R(r) \in(\bar{R}, r)$, and some $c>0$. Hence

$$
\left(\operatorname{Sup}_{B_{R}} u\right)\left(\int_{\bar{R}}^{r} \frac{d t}{\left(\operatorname{vol}\left(B_{t}\right)\right)^{1 / \delta}}\right)^{-1}
$$

is lower bounded away from zero, contradicting (3.3).

\section{Further comments.}

We consider now the case of the $p$-Laplacian. In this setting the structural condition (0.1) is satisfied with $A=1$, and $\delta=p-1, p>1$ and Theorem 3 yields:

Corollary. Let $(M,\langle\rangle)$ be a complete manifold and $r(x)=\operatorname{dist}_{(M,\langle\rangle)}(x, o)$. Suppose that for some $n>1$

$$
\limsup _{r \rightarrow+\infty} \frac{\operatorname{vol}\left(\partial B_{r}(o)\right)}{r^{n-1}}<+\infty .
$$

Let $u \in \mathcal{C}^{2}(M)$ be a p-subharmonic function on $M$ with $p \geq n$. Then $u$ is constant provided either

$$
\liminf _{r \rightarrow+\infty} \frac{\operatorname{Sup}_{r} u}{\log r}=0 \quad \text { if } p=n
$$


or

$$
\liminf _{r \rightarrow+\infty} \frac{\operatorname{Sup}_{r} u}{r^{\frac{p-n}{p-1}}}=0 \quad \text { if } p>n .
$$

We note that in (5.2) and (5.3) we have substituted Sup $u$ with Sup $u$ with the aid of the maximum principle.

This corollary compares directly with the main result in $[\mathbf{R S V}]$, where, with a different technique, we obtain the same conclusion relaxing (5.2) and (5.3) respectively to $\operatorname{Sup} u=O(\log r)$ and $\operatorname{Sup} u=O\left(r^{\frac{p-n}{p-1}}\right)$ as $r \rightarrow+\infty$, $\partial B_{r}$

but strengthening (5.1) to $\lim _{r \rightarrow+\infty} \frac{\operatorname{vol}\left(B_{r}(o)\right)}{r^{n}}=0$.

Next, we show sharpness of the above corollary. Considerations similar to those presented below can be developped for other cases of Theorem 3; notably for the mean curvature operator. We have chosen to consider the $p$-Laplacian for the sake of simplicity.

Next, let $\sigma \in \mathcal{C}^{\infty}([0,+\infty))$ be positive on $(0,+\infty)$ and such that

$$
\sigma(t)=t \quad \text { on }[0,1] .
$$

On $\mathbb{R}^{m} \backslash\{0\}=(0,+\infty) \times S^{m-1}$ we define the metric

$$
\langle,\rangle=d r^{2}+\sigma(r)^{2} d \vartheta^{2}
$$

with $d \vartheta^{2}$ the standard metric on $S^{m-1}$. Because of (5.4) we extend (5.5) to a smooth complete metric on $\mathbb{R}^{m}$.

Let $a(t)$ be a smooth non-negative function on $[0,+\infty)$ satisfying

$$
a(t)=\left\{\begin{array}{ll}
1 & \text { on }[0,1] \text { for } 1<p<2 \\
0 & \text { on }[2,+\infty)
\end{array} ; \quad a(t)= \begin{cases}t^{p-2} & \text { on }[0,1] \text { for } p \geq 2 \\
0 & \text { on }[2,+\infty) .\end{cases}\right.
$$

For $p>1$ we define the non-negative function

$$
u(x)=\int_{0}^{r(x)} \sigma(t)^{-\frac{m-1}{p-1}}\left(\int_{0}^{t} a(s) \sigma(s)^{m-1} d s\right)^{\frac{1}{p-1}} d t .
$$

It is an easy matter to verify that $u \in \mathcal{C}^{2}\left(\mathbb{R}^{m}\right)$ and

$$
\operatorname{div}\left(|\nabla u|^{p-2} \nabla u\right)=a(r(x)) \quad \text { on } \mathbb{R}^{m} .
$$

Thus $u$ is non-constant and $p$-subharmonic. We let $\epsilon>0$ and for $p \geq m$ we choose

$$
\sigma(t)=t^{\frac{p-1}{m-1}}(\log t)^{\frac{p-1+\epsilon}{m-1}} \quad \text { on }[2,+\infty) .
$$

Fix $p \geq m$. It is easily seen that $u(x)$ is bounded, so that (5.2) and (5.3) are satisfied. But

$$
\frac{1}{r^{m-1}} \operatorname{vol}\left(\partial B_{r}\right) \sim r^{p-m}(\log t)^{p-1+\epsilon} \rightarrow+\infty
$$


as $r \rightarrow+\infty$ so that (5.1) is violated.

Next, let

$$
\sigma(t)=t \quad \text { on }[0,+\infty)
$$

so that $\langle$,$\rangle is the canonical flat metric on \mathbb{R}^{m}$. Condition (5.1) is met, but the function $u$ defined above satisfies

$$
\begin{cases}u(x) \sim \log |x| & \text { as } \quad|x| \rightarrow+\infty \text { if } p=m \\ u(x) \sim c|x|^{\frac{p-m}{p-1}} & \text { as }|x| \rightarrow+\infty \text { if } p>m\end{cases}
$$

for some constant $c>0$. Thus conditions (5.2) and (5.3) barely fail to be met.

Remark. If the function $\phi$ satisfies $(0.1)$ on a bounded interval $[0, \alpha)$ and we "a priori" know that $|\nabla u|<\alpha$, the conclusions of Theorem A are still valid.

For instance, the choice

$$
\phi(t)=t\left(1-\frac{\gamma-1}{2} t^{2}\right)^{\frac{1}{\gamma-1}}, \gamma>1
$$

of the left hand side of (0.2) yields the operator describing the equation of continuity in gas dynamics. In this latter instance, the validity of Theorem $\mathrm{A}$ is guaranteed by the requirement

$$
|\nabla u|<\sqrt{\frac{2}{\gamma-1}} \quad \text { on } M
$$

\section{References}

[CY] S.Y. Cheng and S.T. Yau, Differential equations on Riemannian manifolds and their geometric applications, Comm. Pure Appl. Math., 28 (1975), 333-354.

[C] S.S. Chern, On the curvature of a piece of hypersurface in Euclidean space, Abh. Math. Sem. Univ. Hamburg, 29 (1965), 77-91.

[CK] P. Collin and R. Krust, Le problème de Dirichlet pour l'equation des surfaces minimales sur des domaines non bornés, Bull. Soc. Math. France, 119 (1991), 443-462.

[F] H. Flanders, Remark on mean curvature, J. London Math. Soc., 41 (1966), 364-366.

$[\mathrm{H}] \quad$ E. Heinz, Über Flächen mit eineindeutiger Projektion auf eine Ebene, deren Krümmungen durch Ungleichungen eingeschränkt sind, Math. Ann., 129 (1955), 451-454.

[H1] J.F. Hwang, A uniquess theorem for the minimal surface equation, Pac. J. Math., 176 (1996), 357-364.

[H2] Comparison principles and theorems for prescribed mean curvature equations in unbounded domains, Ann. Scuola Norm. Sup., Pisa, 15 (1988), 341-355.

[K1] L. Karp, Differential inequalities on complete Riemannian manifolds, Math. Ann., 272 (1985), 449-459. 
[K2] _ Subharmonic functions on real and complex manifolds, Math. Z., 179 (1982), 535-554.

[M] V.M. Miklyukov, On a new approach to Bernstein's theorem and related questions for equations of minimal surface type, Math. Sb., 108 (1979), 268-289.

[RSV] M. Rigoli, M. Salvatori and M. Vignati, Volume growth and p-subharmonic functions on complete manifolds, Math. Z., 227 (1998), 367-375.

[S] I.M.C. Salavessa, Graphs with parallel mean curvature, Proc. Amer. Math. Soc., 107 (1989), 449-458.

Received June 11, 1998 and revised November 24, 1998.

Università Di Milano-BicocCA

20126 Milano, Italy

E-mail address: rigoli@matapp.unimib.it

Università DEGLi Studi Di Milano

VIA SALDINI 50

20133 Milano

ITALY

E-mail address: maura.salvatori@mat.unimi.it

Università DEGLi Studi di Milano

VIA SALDINI 50

20133 Milano

ITALY

E-mail address: marco.vignati@mat.unimi.it 